\title{
- \\ NASA'S LIVING WITH A STAR PROGRAM: THE GEOSPACE MISSION CONCEPT
}

AIAA-2002-0086

\author{
Janet L. Barth, Discipline Scientist, NASA/Goddard, Greenbelt, MD \\ Barbara Giles, Discipline Scientist, NASA/Goddard, Greenbelt, MD \\ Lawrence Zanetti, Senior Project Scientist, Johns Hopkins University/Applied Physics Laboratory, Laurel, MD \\ James Spann, Program Scientist, NASA/Headquarters, Washington, DC
}

\begin{abstract}
NASA has initiated the Living with a Star Program (LWS) to develop the scientific understanding to address the aspects of the Connected Sun-Earth system that affect life and society. A goal of the program is to bridge the gap between science, engineering, and user application communities. This will enable future science, operational, and commercial objectives in space and atmospheric environments by improving engineering approaches to the accommodation and/or mitigation of the effects of solar variability on technological systems. Three program elements are the Science Missions; a Theory, Modeling, and Data Analysis program; and a Space Environment Testbeds program. Because many of the effects of solar variability on humanity are observed in Geospace regions of space, the science research for all three elements of the LWS Program have significant components in Geospace regions.
\end{abstract}

\section{INTRODUCTION}

The Sun, the astronomical object most significant to humanity, affects the entire geospace region. Because of the consequences to the Earth of the Sun's dynamic behavior and the rapidly expanding utilization of this region for human activities, a thorough understanding of the Sun's effects is becoming increasingly essential. The Sun radiates both as electromagnetic energy and as fast-moving electrically charged particles. The electromagnetic radiation across a broad spectrum of wavelengths originates from the photosphere, the Sun's surface. This energy proceeds unimpeded directly from the Sun to the Earth's atmosphere, with a majority reaching the surface of the Earth.

The effects of the Sun-Earth interaction are far-

Copyright $\odot 2002$ by the American Institute of Aeronautics and Astronautics, Inc. No copyright is asserted in the United States under Title 17, U.S. Code. The U.S. Government has a royalty-free license to exercise all rights under the copyright claimed herein for Governmental purposes. All other rights are reserved by the copyright owner. reaching and have impacts on our technological systems, humans in space and on some aircraft routes, and terrestrial climate change [1]. With the exception of spacecraft missions outside of the Earth's magnetosphere, most of the impacts of solar variability on humanity are observed in geospace regions.

Minimizing the impacts of the effects on technology, life, and society requires that we improve the scientific understanding for how and why the changes occur on the Sun as well as how the Sun and Earth interact as a system. We can then use the results to enable engineering applications to improve capabilities for future missions.

NASA initiated the LWS Program in its Office of Space Science (OSS) to develop the scientific understanding to address the aspects of the connected Sun-Earth system that affect life and society. Three fundamental questions will be addressed to develop this understanding, as follows:

- How and why does the Sun vary?

- How do the Earth and other planets respond? -

- What are the implications for humanity?

The LWS Program objectives are based upon these questions and are as follows:

- Understand solar variability and its effects on the space and Earth environments with an ultimate goal of a reliable predictive capability of solar variability;

- Obtain scientific knowledge relevant to mitigation or accommodation of undesirable effects of solar variability on humans and human technology; and,

- Understand how solar variability affects hardware performance and operations in space.

The formulation of the LWS Program architecture and implementation plan is currently in progress with inputs from the science and engineering communities. There are plans for two groups of science spacecraft: (a) solar dynamics elements that observe the Sun and track disturbances originating there and (b) geospace elements located between the Sun and the Earth and around the Earth to measure downstream effects the geospace response to changes in solar activity. Plans also include a data analysis component to improve 
scientific understanding, and a series of Space Environment Testbeds (SET) to provide infusion of the improved understanding to the design and operations of future missions. The LWS Program is developing partnerships with other US and International agencies and industry to augment existing capabilities.

The Geospace Mission Concept includes all of the implementation elements discussed above. The Geospace Mission Definition Team (GMDT) is formulating the geospace science missions at this time. The final report, which will be used to generate requirements for the space segments, is due in March 2002. The purpose of this paper is to describe the overall Geospace Mission Plan of the LWS Program, including some of the preliminary results of the GMDT,

\section{THE LIVING WITH A STAR PROGRAM ARCHITECTURE}

The LWS Program requirements were developed using a series of community workshops [2], results of the Space Science strategic planning process $[3,4]$, interagency discussions, and report by the National Space Weather Program [5], and National Research Council/National Academy of Sciences [6]. Participants were from U.S. industry, universities, and government agencies. They are customers for the understanding and knowledge resulting from the Program. The result is the four program elements:
- Science Missions,

- Theory, Modeling, and Data Analysis,

- Space Environment Testbeds, and

- Evolution of established and expanded partnerships.

These elements are integrated into an LWS Program in the OSS's Sun Earth Connection (SEC) Division and implemented through an LWS Program Office at the NASA Goddard Space Flight Center. They are described in the following sections of this paper. The SEC science community provides advice to the OSS through the Sun Earth Connection Advisory Subcommittee (SECAS), a subcommittee of the Space Science Advisory Committee [7]. The SET Steering Committee provides advice to the SET and the SECAS. A schematic of this LWS program architecture is given in Fig. 1.

The Science Missions are a distributed network of research spacecraft to quantify the physics, dynamics, and behavior of the Sun-Earth connected system through the range of conditions occurring during the eleven-year solar cycle. The science missions gather basic science data to achieve the understanding of the connected Sun-Earth system.

Data mining and analysis in a Theory, Modeling, and Data Analysis (TMDA) component will improve knowledge of space environmental conditions and variations over the solar cycle, develop new techniques

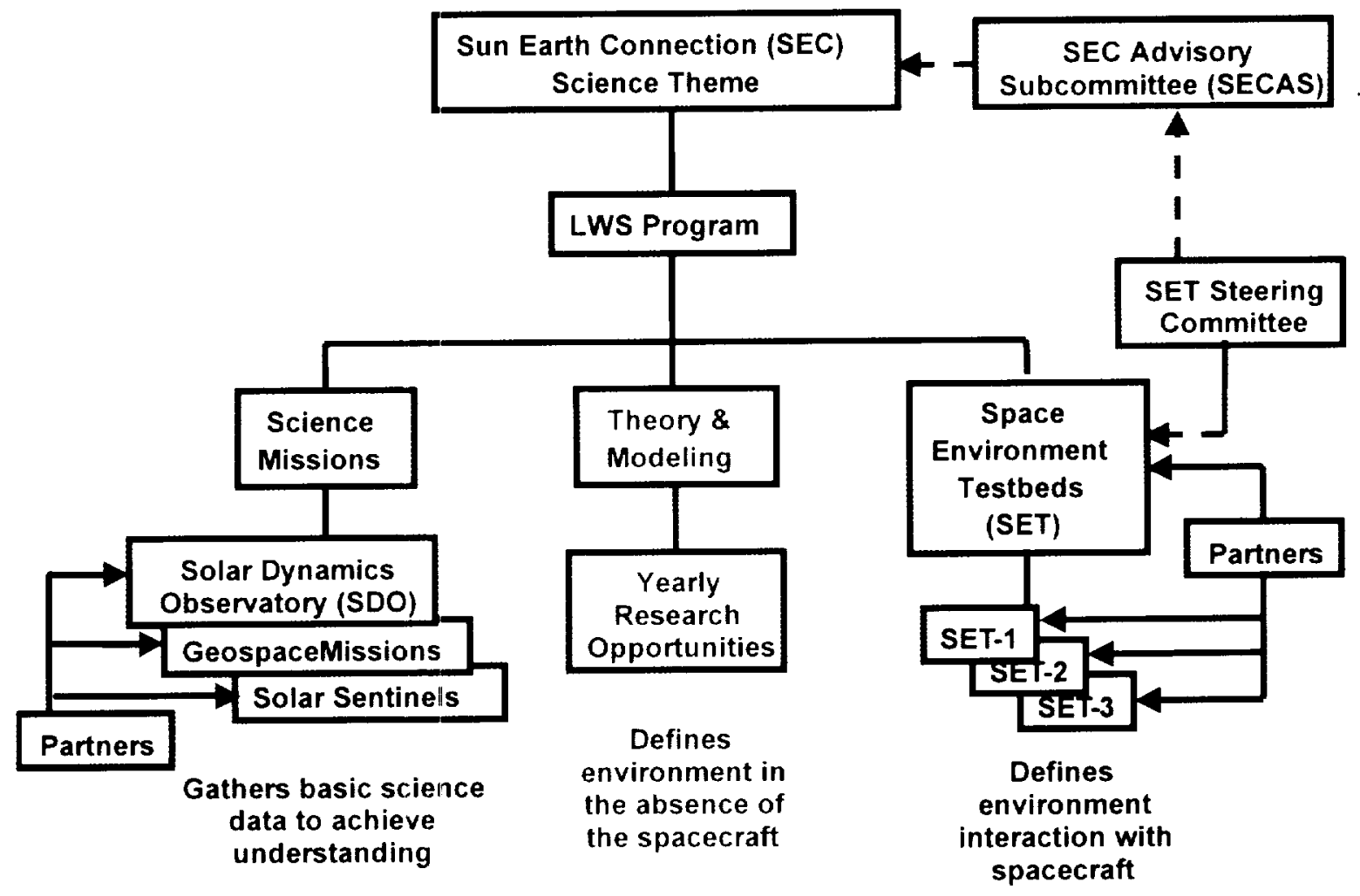

Fig. 1: This figure illustrates the program architecture of the LWS program. 
and models for predicting solar/geospace disturbances, and develop cost-effective techniques for assimilating data from networks of spacecraft. The TMDA defines the environment in the absence of a spacecraft.

The sequence of SET [1] will reduce the uncertainty in the space environment definition as functions of location and time in the solar cycle and will characterize the space environment effects on space hardware. The SET tasks are restricted to the interactions that change with solar variability.

\section{THE GEOSPACE CONCEPT}

All of the elements of the LWS Program architecture have geospace components so that the LWS Program can adequately address the scientific problems in the geospace region. Each of the elements as they apply to geospace will be discussed in the next section.

\section{GEOSPACE SCIENCE MISSIONS}

Fig. 2 shows one concept for the Science Mission network. The distributed network of spacecraft will provide continuous observations of Sun-Earth System and has two components:

- Solar Dynamics Network observing Sun and tracking disturbances from Sun to Earth

- Geospace Dynamics Network observing changes in the near-Earth environment
The first missions planned in the Solar Dynamics Network are the Solar Dynamics Observatory (SDO) [8] and Solar Sentinels. The SDO will observe the Sun's dynamics and help us understand the nature and source of variations, from the stellar core to the turbulent solar atmosphere. Sentinels will provide a global view of the heliosphere and describe the transition and evolution of eruptions and flares from the Sun to Earth.

The goal of the Geospace science missions is to increase scientific understanding of how the Earth's ionosphere and magnetosphere respond to changes due to solar variability. There are two focus areas:

- Radiation belts: The chain of processes that populate, energize, transport, and cause losses of the radiation belt particles

- Ionosphere: The main contributions to the heating and ionization of the ionosphere and thermosphere; solar EUV, auroral precipitation, Joule heating, and the ionosphere portion of the electric current system

The GMDT is developing requirements for science missions that will determine how the geospace region behaves as a system and to discover, understand and model the connection between solar phenomena and geospace response. (See Fig. 3). The NASA's SolarTerrestrial Probe (STP) Program will also support the science requirements of geospace by discovering and understanding basic science phenomena.

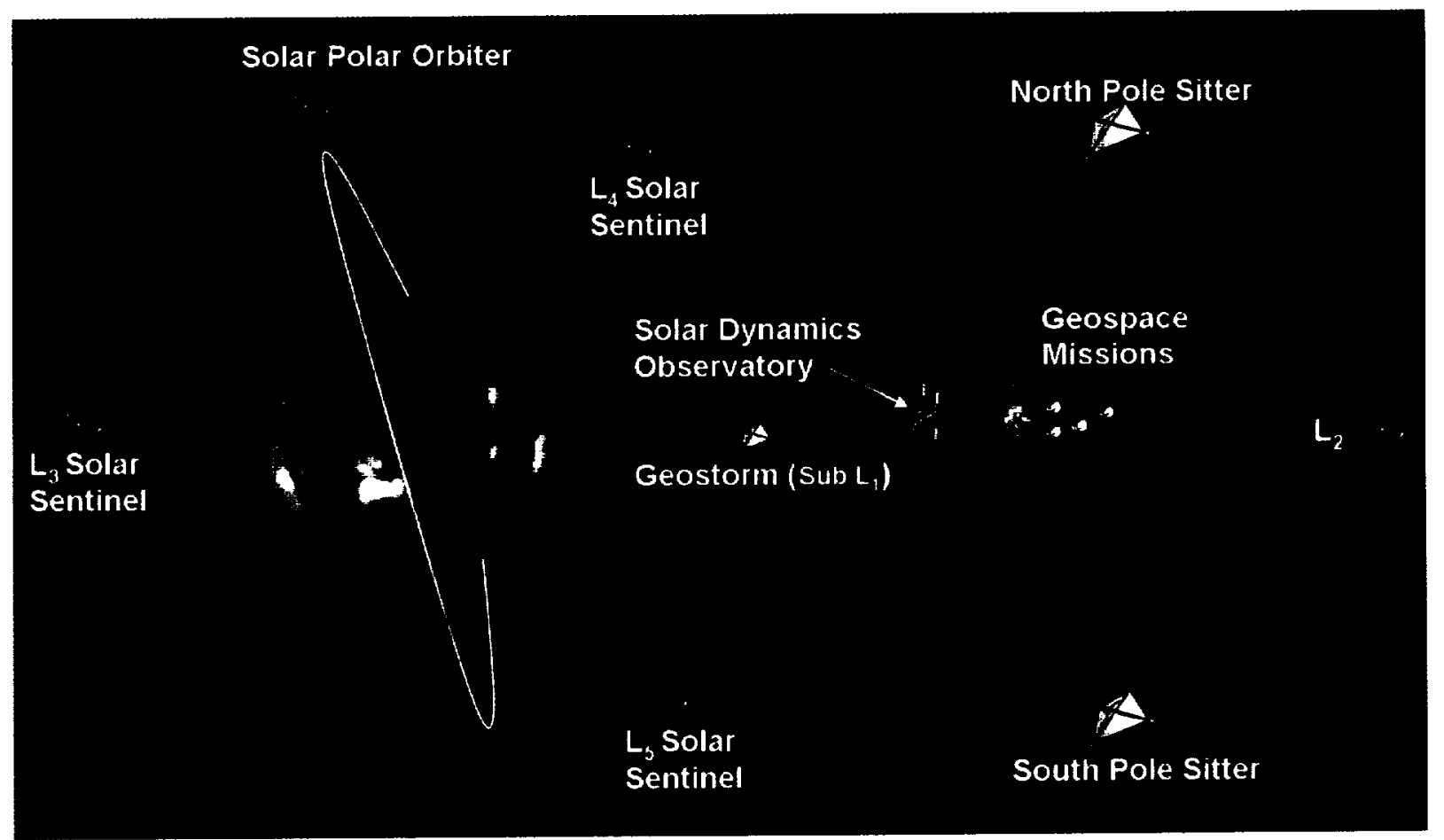

Fig. 2: A concept for the LWS science network 
A preliminary implementation plan was formulated to achieve the goals of the geospace science missions. The plan envisioned a high level of partnering to provide adequate coverage within the LWS Program resources. The approach has four components:

- The Geospace Missions Network

- Missions of Opportunity

- Leveraged Programs

- Instrument Development Program

The Geospace Missions Network is the primary science component of the Geospace science mission plan. The network will consist of several small NASA missions flown concurrently in geospace regions. The three elements of the network are 1) a radiation environment mission with spacecraft in low inclination, elliptical orbits, one being a geostationary transfer (GTO) orbit, 2) an ionosphere mission with spacecraft in low earth orbits (LEOs), and 3) a geospace imaging mission with a single spacecraft in a elliptical polar orbit. The launch of the major component is planned for 2008 so that the Solar Dynamics and Geospace Dynamics components of the research network will be in place for the next solar maximum.
Missions of opportunity are key instrumentation platforms to provide additional observations. The purpose is to characterize the extremes of the geospace environment outside of the primary Geospace Missions Network. Studies are underway to include instrumentation on the LWS SET and Solar Dynamics Observatory and to work with the missions of international space weather programs. Launches are planned throughout the solar cycle.

Leveraged missions also play a role in the Geospace science missions by providing measurements from existing platforms. These missions could include Communication/Navigation Outage Forecasting System (C/NOFS), Defense Meteorological Satellite Program (DMSP), Geosynchronous Operational Environmental Satellites (GOES), Polar-orbiting Operational Environmental Satellite (POES), Global Positioning System (GPS), National Polar-orbiting Operational Environmental Satellite System (NPOESS), and Meteorological Operation (METOP) satellites.

Fig. 4 shows the geospace coverage that is possible with the three mission components. All of the three elements require the development of resource efficient instruments.

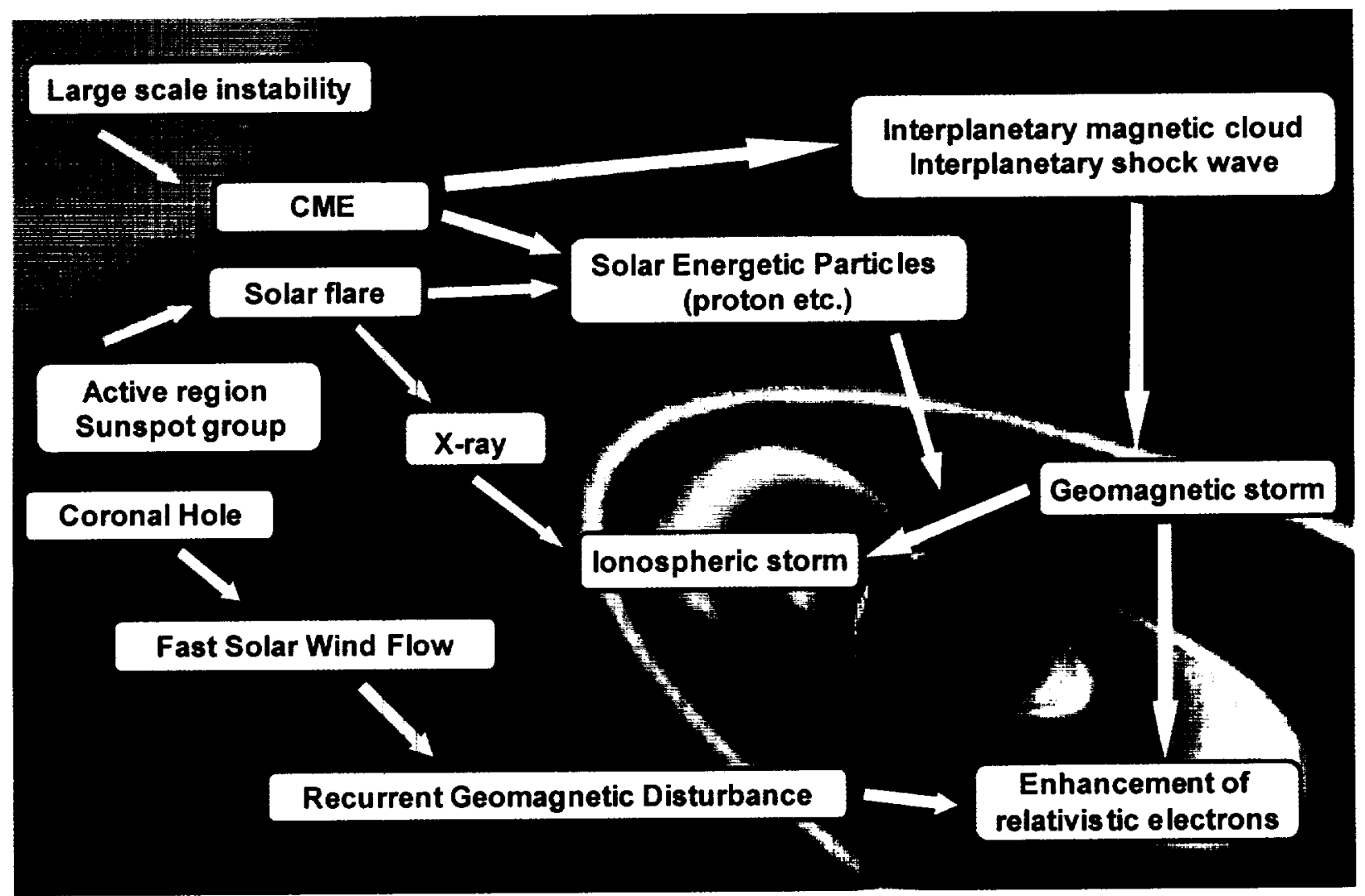

Fig. 3: This figure shows some of the important connection processes that require scientific understanding. 


\section{LWS THEORY, MODELING, AND DATA}

\section{ANALYSIS}

The Theory, Modeling, and Data Analysis element of the LWS Program includes investigations in geospace regions. The investigations are ground based set of analyses that are performed using data from past and present space missions, including the future LWS Science Missions. The types of investigations are:

- Develop new instrument techniques, models, and concepts for investigating solar and geospace disturbances

- Enhance the understanding of the role of solar influences on terrestrial global climate

- Improve scientific knowledge of space environment conditions and variations over the solar cycle

- Develop models and concepts for the infrastructure of future LW'S missions

Investigation topics must be research that address SunEarth Connection processes that have direct impact on life and society. Examples include but are not limited to: understanding and prediction of solar activity, acceleration and transport of solar energetic particles, refinement of the understanding of space weather, the role of solar variability in terrestrial climate change, and development environment specification models with better predictive capability to support technological systems design, operations risk management, and on-orbit operations.

The TMDA program is implemented through peerreviewed competition. Participants may be from industry, universities, government agencies, and international partners. Research opportunities are announced by NASA/Headquarters through the Research Opportunities in Space Science (ROSS). The first announcement was in 2000 with proposals awarded in 2001. Of the sixty awards in 2000 , at least $50 \%$ directly addressed issues related to the geospace region [9]. The ROSS proposals for awards in 2002 were due in Sept. 2001.

\section{LWS SPACE ENVIRONMENT TESTBEDS}

The purpose of the SET program is to perform applications research on space environment interactions with instruments and spacecraft. The objective is to increase the capability to design and operate "all weather" space systems by improving the engineering approach to accommodate and/or mitigate the effects of solar variability. The program bridges the gap between

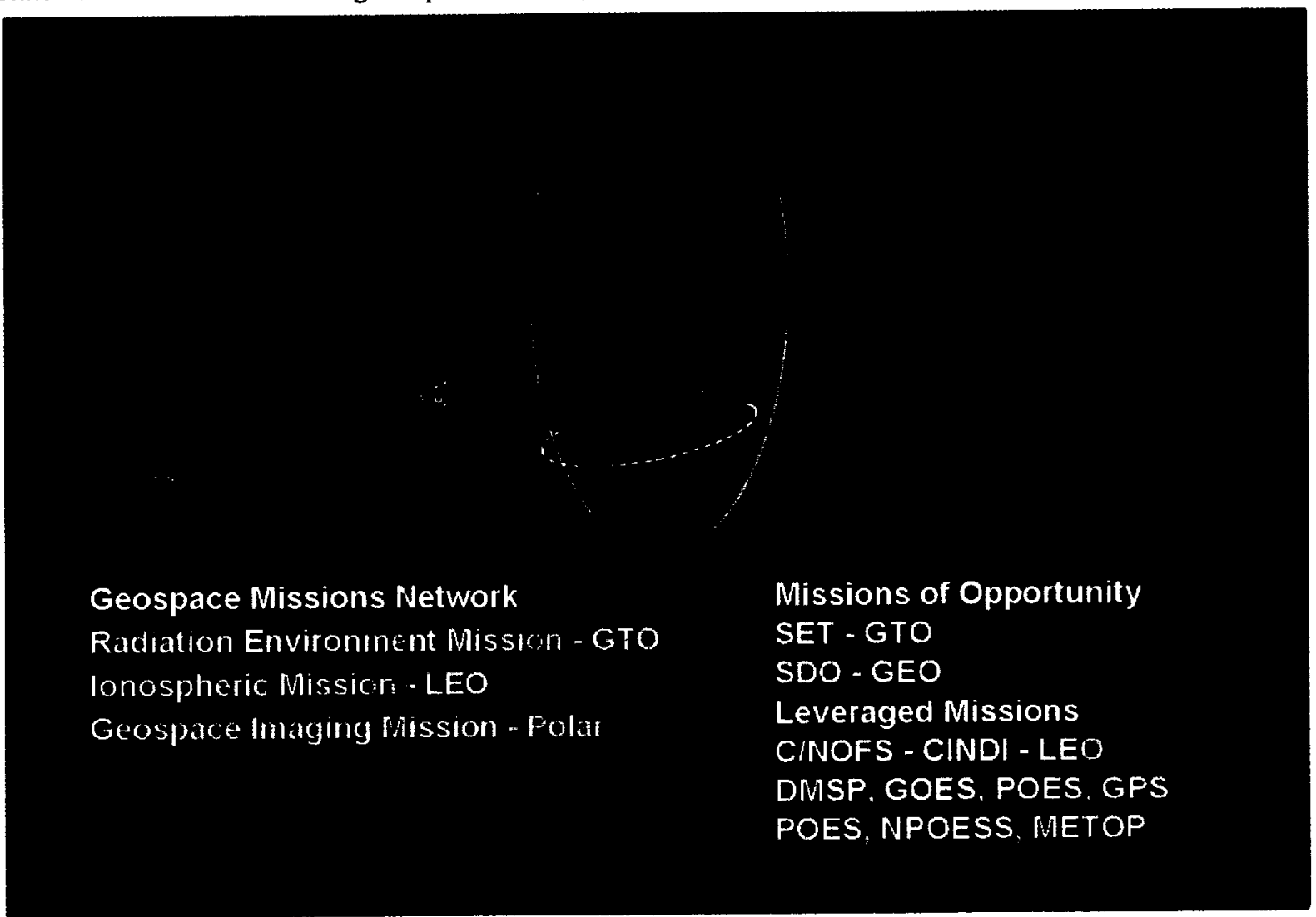

Fig. 4: Possible mission approach for the Geospace Dynamics Network 
the science, engineering, and user application communities by directly measuring the effects of solar variability on technological systems, particularly the sudden changes induced by space storms. Fig. 5 shows the direct infusion path from new results from science missions to engineering products that the LWS Program architecture enables.

The experiment investigations will include microelectronics technologies; shielding properties and degradation of materials; detectors, such as charge coupled devices (CCDs) and active pixel sensors (APS); and spacecraft charging effects. The experiments can fly on the SET experiment carriers or on flights of opportunity on other commercial, international, or government missions. The experiment payloads of the SETs will determine the orbit requirements, however, it is anticipated that all of the SETs will fly in geospace regions. The preferred orbits are high inclination/low earth and geostationary transfer because they provide sampling of atmospheric, plasma, debris and meteoroid, and radiation environments.

The SET program implementation plan is to hold planning and progress workshops, fund NASA Research Announcements (NRAs), design and develop an experiment carrier, and fly on-board environment monitors appropriate for the experiments. It is also important to establish partnerships with industry, international agencies, and US agencies to fully implement the program.

The SET concept contains three types of investigation components based upon customer requirements: collateral environments measuring instruments, box experiments, and board experiments. Collateral environment measurements are performed to support the flight validation and performance characterization experiments. Box experiments are independent, self-contained experiments in separate packages. They interface directly to the testbed for power and data. Board experiments are modular card experiments that connect to a central board through a simple and common interface for analysis, storage and transfer of data, and power conditioning.

A concept for a common experiment interface to a testbed and a simple interface between a testbed and a

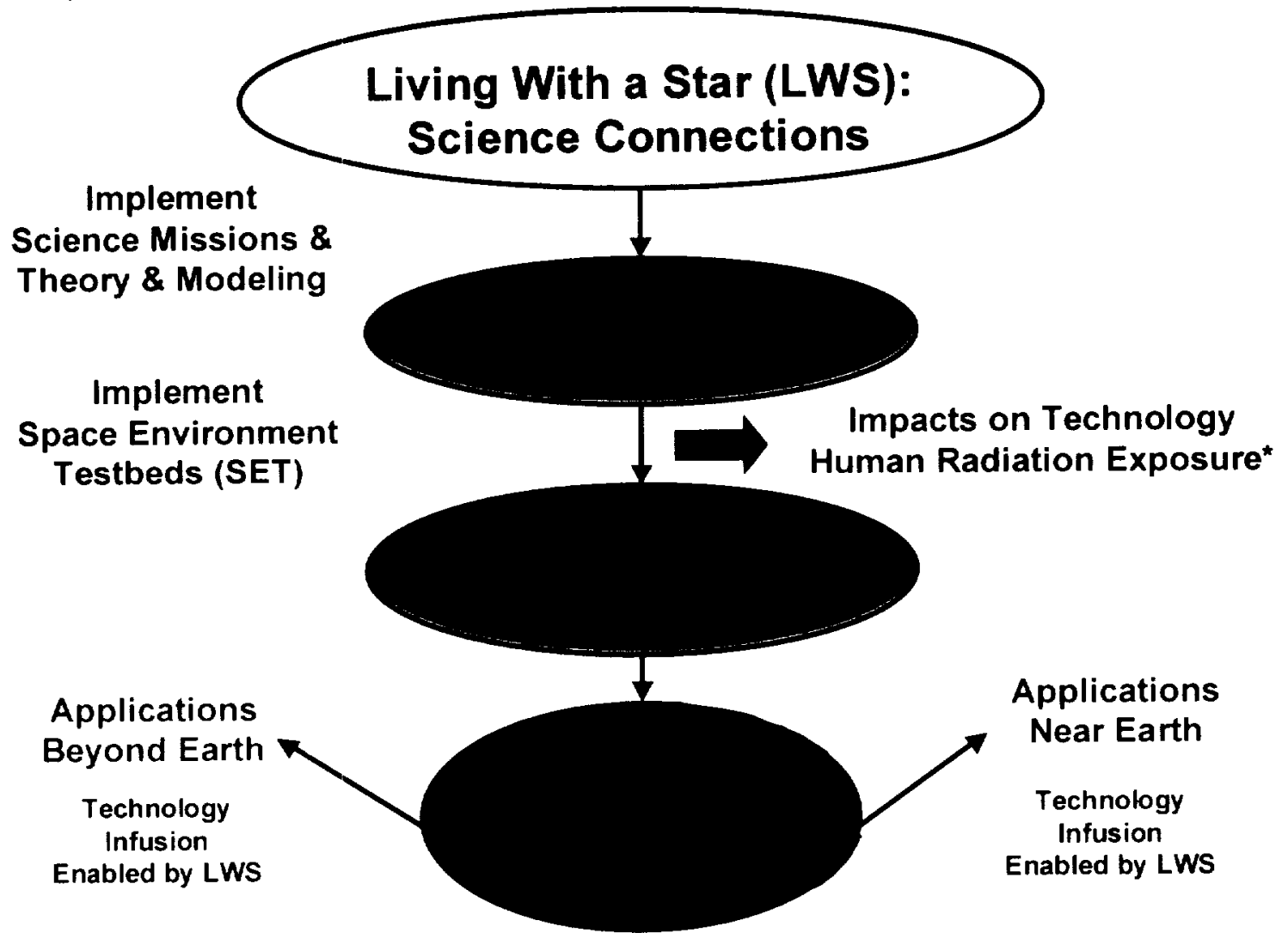

Fig. 5: The direct connection between science missions and engineering applications 
host spacecraft has been developed and is shown in Fig. 6. In the figure, the microelectronics testbed is shown as an embedded testbed that interfaces to the central SET testbed and contains card experiments. The testbed provides a central processing unit (CPU) to the experiments. Stand-alone (box) and card experiments are shown.

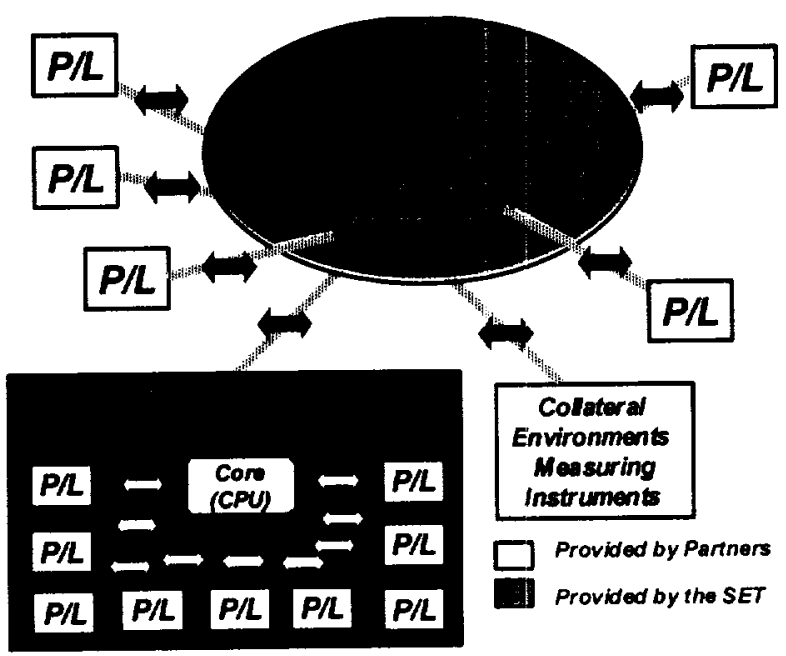

Fig. 6: The SET interface concept

\section{THE LWS PROGRAM TIMELINE}

The LWS Program is a continuously funded program that will be implemented throughout the phases of the solar cycle. The Science Missions (SDO, Solar Sentinels, Geospace) will be in place for the next solar maximum. The SDO launch is planned for 2007 and the Geospace launch is planned for 2008. The SDO Science Announcement of Opportunity $(\mathrm{AO})$ will be issued in January 2002, and the Geospace science mission plans to release a NASA Research Announcement for instrument development in 2002.

The SET program awarded research investigations for data mining research in December 2001, and the first research opportunity for experiment development will be in February 2002. The launches are planned on 2-year centers with the first in 2005/2006. A "pathfinder mission" is being developed for 2004.

The TMDA program issues yearly AOs through the Research Opportunities in Space Science. The first TMDA awards were in 2001, and proposals have been accepted for the 2002 awards.

\section{SUMMARY}

NASA's Living with a Star program is a multidiscipline science research program that will take an integrated approach to understanding solar variability and its effects. We have shown how the LWS program elements (the science missions, theory and modeling, space environment testbeds, and program partnering) are being designed and implemented to understand the effects of solar variability in geospace regions. The program addresses pure and applied science.

\section{REFERENCES}

[1] J. L. Barth, K. A. LaBel, D. A. Brewer, G. Withbroe, W. Kauffman, "The Living With A Star Program: NASA's Role in Assuring Performance in Space and Atmospheric Environments," Presented at AIAA Aerospace Conference, AIAA2001-0235, Reno, NV, January 8-11, 2001.

[2] Anon., Living with a Star (LWS) Pre-Formulation Study, Internet address http://lws.gsfc.nasa.gov/lws.htm, NASA Goddard Space Flight Center, Greenbelt, MD, Aug. 2000.

[3] Anon., The Space Science Enterprise Strategic Plan, Doc. No. NP-2000-08-258-HQ. NASA Headquarters, Washington, DC, Nov. 2000. Avail. http://spacescience.nasa.gov.

[4] Anon., Sun-Earth Connection Roadmap: Strategic Planning for 2005-2025. NASA Headquarters, Washington, DC, Jan. 2000. Avail. http://www.lmsal.com/sec/.

[5] Anon., National Space Weather Program: Implementation Plan. Doc. No. FCM-P31-1997, Office of the Federal Coordinator for Meteorological Services and Supporting Research, Washington, DC, Jan. 1997. Avail. http://www.ofcm.gov/nswp-ip/text/cover.htm.

[6] National Research Council, Radiation and the International Space Station: Recommendations to Reduce Risk. Space Studies Board and Board on Atmospheric Sciences and Climate, National Academy Press, Washington, DC, 2000.

[7] See http://www.hq.nasa.gov/office/ codez/new/ for the charter of the Space Science Advisory Committee.

[8] See http://lws.gsfc.nasa.gov/lws_missions_sdo.htm for the results from the SDO Science Definition Team.

[9] See http://research.hq.nasa.gov/code_s/nra/current/ NRA-00-OSS0-1/LWSabstracts.html for information on the TMDA 2001 awards. 